\title{
EFFECT OF THE LOCAL DAMAGE AND PROFILE ERROR OF THE GEAR ON THE DRIVETRAIN DYNAMIC RESPONSE
}

\author{
Ahmed Ghorbel, Bacem Zghal, Moez Abdennadher, \\ LASSAD WALHA, MOHAMED HADDAR \\ Mechanics, Modelling and Manufacturing Laboratory (LA2MP), Mechanical Engineering Department, National Engineers \\ School of Sfax, Sfax, Tunisia \\ e-mail: gh.ahmed.enis@gmail.com
}

\begin{abstract}
The dynamic modeling of vibration of a drivetrain is used for increasing our information about vibration generating mechanisms, especially in the presence of some kind of gear faults. This paper describes a research work on the automotive driveline modeling, vibration analysis, and the effect of gear defects on the dynamic behavior of the system. Firstly, main drivetrain components including the engine, clutch, single stage spur gearbox and disc brake are modeled, respectively. The nonlinear dynamic model is simulated by a thirteen degrees of freedom (DOF) system and the nonlinear function is due to the dry friction path. Secondly, two types of defects are modeled and introduced into the spur gear system; local damage and profile error. Then, the nonlinear equations of motion are solved by the numerical Runge Kutta method and a comparative study of the dynamic behavior of the system in healthy and defected cases is discussed for each fault type. The influence of the defects on the vibration response is presented in the time and frequency domain. Finally, analysis of the two defects together is presented.
\end{abstract}

Keywords: dynamic behavior, clutch, gear, brake disc, gear local damage, gear profile error

\section{Introduction}

All conventional automotive vehicles must have drivetrains. The objective of the drivetrain is to transfer the power developed in a thermal engine to driving wheels. Vibro-impacts in an automotive driveline system are a critical concern to vehicle manufactures based on noise, vibration and reliability considerations.

In this context and in order to determine the vibratory behavior, the modeling and studying a drivetrain system is an important research field addressed by many researchers. Bemporad et al. (2001) proposed some simple models with two masses where the first represented the engine and the second the vehicle which included the powertrain, clutch and shafts. Templin and Egardt (2009) investigated a torsional drivetrain model with only two degrees of freedom. A more detailed model was developed by Walha et al. (2011) to study the effect of the eccentricity defect on the dynamic behavior of a coupled clutch-helical two stage gear system. Brancati et al. (2007) intend to modeled and investigate the effect of oil damping on the dynamic behavior where the considered model was constituted by a flywheel, clutch and gear pairs of an automotive transmission. Wu and Guangqiang (2016) developed a torsional driveline model to analyze torsional vibration and reduce the gear rattle. Ghorbel et al. (2017) proposed a linear model with 22 DOFs including the main subsystems of an automotive drivetrain (engine, clutch, two helical gear stage and disc brake) to investigate their dynamic behavior and modal properties.

A nonlinear torsional model of the clutch was developed by Xue-Lai et al. (2016) for investigating the influence of each parameter on noise generation. Gaillard and Singh (2000) proposed five models of an automotive clutch with the dry friction path and investigated energy dissipation 
for each model. Driss et al. (2007) presented a clutch model with 11 DOFs which included three types of nonlinearity. Saleh et al. (2015), Krak et al. (2015) investigated multi stage stiffness and hysteresis phenomena with spline nonlinearities in the vibrational behavior of the clutch. Also, the influence of clutch characteristics of the gearbox rattling in different engine conditions was studied.

Concerning gearbox modeling, the literature is rich in analytical and experimental works on gear systems. A detailed model of gear dynamic vibration was investigated in many review papers (Chaari et al., 2008; Howard et al., 2011; Kim et al., 2010; Walha et al., 2009). These models are currently heavily used and can include shaft torsional vibration and bearing stiffness. A gear mechanism may undergo several types of defects. The gear faults can be classified into three types: manufacturing defects (eccentricity of wheels, tooth profile errors, etc.), assembly defects (misalignment, parallelism, etc.) and defects appearing during transmission error. In many earlier gear models, defects or errors have not been taken into account, especially the local damage and profile error. The research work by Rincon et al. (2012) includes different types of faults that may affect the gearing and studies their influence an the dynamic response. Indeed, Fakhfakh et al. (2005) studied the influence of teeth defects of a gear through variation it makes to the mesh stiffness. In another work, Fakhfakh et al. (2006) analyzed the dynamic behavior of two stage gears in presence of tooth manufacturing defects. Divandari et al. (2012) investigated the effect of profile modifications and errors on the gear system dynamic behavior in the presence of tooth localized defects. Detections of defects become important tools to know health of the gearbox. There are various vibration analysis techniques which can be used for this purpose. Time domain frequency domain techniques and time frequency analysis are the methods that can be employed for gearbox diagnostics (Abouel-seoud et al., 2012; Aherwar and Khalid, 2012; Kobra et al., 2010). In this paper, the time domain response and the Fast Fourier Transform (FFT) are used to investigate the effect of gear faults on the drivetrain dynamic behavior.

A wide range of vehicles are equipped with disc brakes which offer many advantages over drum brakes. The experimental observations of Fosberry and Holubecki (1961) showed that the vibrations of the disc were much larger than those of the caliper of about $20 \mu \mathrm{m}$. Ahmed (2011) developed a detailed theoretical model of the disc brake to reduce squeal phenomena. In another work, a theoretical model was proposed in order to analyze the influence of the disc brake friction model of the dynamic response of one-stage spur gear transmission (Khabou et al., 2014).

It should be noted that although the modeling and analysis of the drivetrain dynamic behavior presented in the paper already exist in the literature, few results actually address the dynamic response which includes the influence of gear defects on a coupled system. In this article, we propose a new nonlinear dynamic model of a drivetrain system that contains thirteen degrees of freedom. Two typical defects of the gear (local damage and profile error) are introduced to study their effect on the dynamic response.

\section{Dynamic drivetrain model}

In this Section, a simple drivetrain model is presented in Fig. 1. This model is composed of three blocks; clutch, single spur gear stage and disc brake, which are supported by three bearings, one for the input, the second for the shaft linking the clutch with the geared system and the third one for the output shaft. The drivetrain system is driven by an engine which transmits torque $T_{e}(t)$ to the input shaft by a coupling. A load torque $T_{d}$ is applied to the brake disc of the output shaft.

Using Fourier series decomposition, the engine torque $T_{e}(t)$ can be written as follows

$$
T_{e}(t)=T_{p}+T_{p m} \cos \left(\frac{n_{c}}{2} \Omega_{e} t+\phi_{p n}\right)
$$


where $n_{c}$ and $\Omega_{e}$ are the number of engine cylinders and the engine rotational speed. $T_{p}, T_{p m}$ are respectively the average amplitude and the amplitude of the harmonic, and $\phi_{p n}$ is the associated phase. To simplify the problem, the phase is supposed to be equal to zero.

The system has thirteen degrees of freedom (DOF) which can be defined as follows:

- Rotation along the $z$ axis of the flywheel and cover $\theta_{1}$, pressure plate $\theta_{2}$, friction disc $\theta_{3}$, clutch hub $\theta_{4}$, pinion $12 \theta_{12}$, pinion $21 \theta_{21}$ and brake disc $\theta_{d}$.

- Translations of the first block (mass $m_{b 1}$ ) composed of the engine, flywheel, cover and pressure plate along the $x$ and $y$ directions. The corresponding DOFs are $x_{1}$ and $y_{1}$.

- Translations of the intermediate block (mass $m_{b 2}$ ) composed of the friction disc, clutch hub and pinion 12 along the $x$ and $y$ directions. The corresponding DOFs are $x_{2}$ and $y_{2}$.

- Translations of the output block (mass $m_{b 3}$ ) composed of wheel 21 and the disc brake along the $x$ and $y$ directions. The corresponding DOFs are $x_{3}$ and $y_{3}$.

- The following inertias are considered: $I_{1}$ for the flywheel and cover, $I_{2}$ for the pressure plate, $I_{3}$ for the friction disc, $I_{4}$ for the clutch hub, $I_{12}$ for pinion 12, $I_{21}$ for wheel 21 and $I_{d}$ for the brake disc.

- The elasticity of three shafts is modelled by torsional stiffnesses, respectively $K_{1}, K_{2}$ and $K_{3}$. Bearings supporting the input, intermediate and output shafts are respectively modelled by linear stiffnesses $k_{x i}$ and $k_{y i}(i=1,2,3)$ acting along the $x$ and $y$ directions.

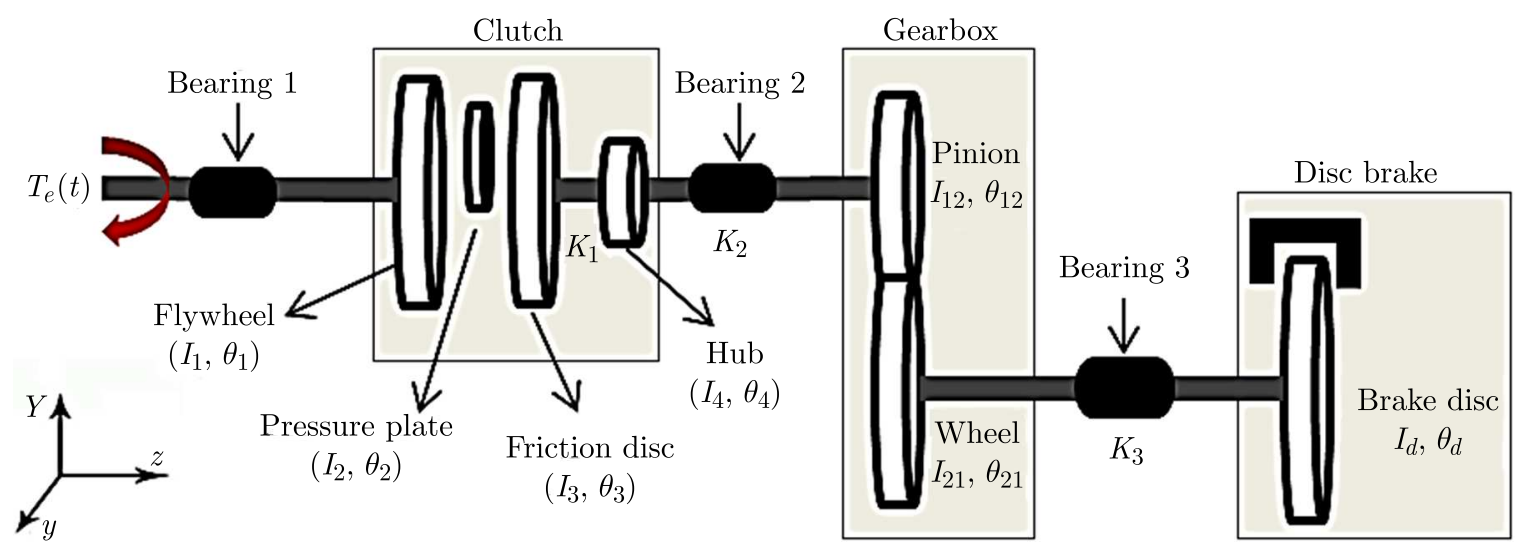

Fig. 1. Vehicle drivetrain model

\subsection{Vehicle clutch modelling}

The clutch is an important component of the car driveline which can play a significant role in filtering vibrations due to fluctuations of the engine torque. The modeling of a vehicle clutch is affected by a torsional model with a nonlinear function, see Fig. 2. This is due to dry friction. The friction torque $\widetilde{T}_{f}(\dot{\delta})$ is a nonlinear function which depends on the relative velocity $\dot{\delta}$, such that $\dot{\delta}_{2}$ is the relative velocity between the pressure plate and the friction disc and $\dot{\delta}_{3}$ describes the relative velocity between the flywheel and the friction disc. $K_{1}$ represents the torsional stiffness of the shaft and $k_{12}$ is torsional stiffness between the cover and the pressing plate.

The equation of the friction torque is

$$
T_{f}(\dot{\delta})=\left(\mu_{D}+\left(\mu_{S}-\mu_{D}\right) \mathrm{e}^{\varepsilon|\dot{\delta}|} \tanh (\sigma \dot{\delta})\right) P A R
$$

where $P$ is the pressure, $A$ is the surface area of contact and $R$ is the mean radius of that surface. $\mu_{s}$ and $\mu_{D}$ are respectively the static and dynamic friction coefficients, $\varepsilon$ is a factor which controls the gradient of exponential decaying and $\sigma$ is the conditioning factor that controls the smoothing level at the discontinuity of this function. The $\varepsilon$ and $\sigma$ values are verified with those by Walha et al. (2011), and they are equal to: $\varepsilon=2$ and $\sigma=50$. 


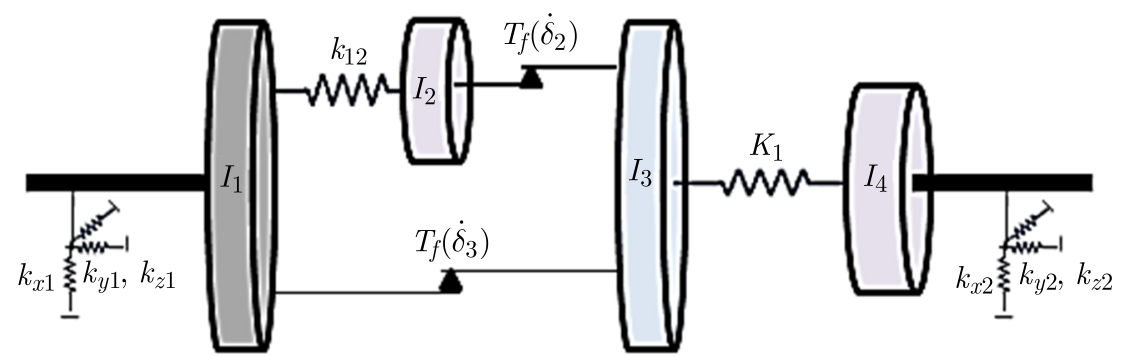

Fig. 2. Dynamic clutch model

\subsection{Spur gear system modelling}

The gearbox mechanism contains a single stage spur gear (Fig. 3). Toothed wheels 12 and 21 are assumed to be rigid bodies and bearings 2 and 3 are modeled by linear stiffness $k_{x i}$ and $k_{y i}$ $(i=2,3)$. A periodic variation of the gear mesh stiffness $k(t)$ is another excitation source to the system and it is generated by the time varying number of teeth in contact. Figure 4 shows the evolution of gear mesh stiffness in the healthy case. The spectrum in this case is composed by the meshing frequency $f_{m}$ and its harmonics.

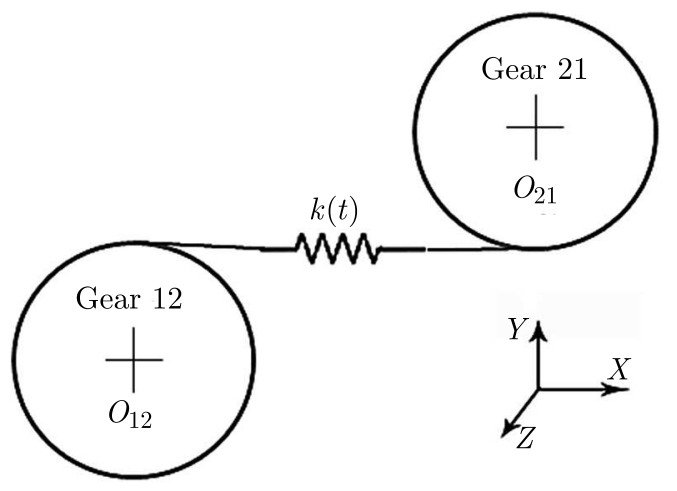

Fig. 3. Single stage spur gear modelling
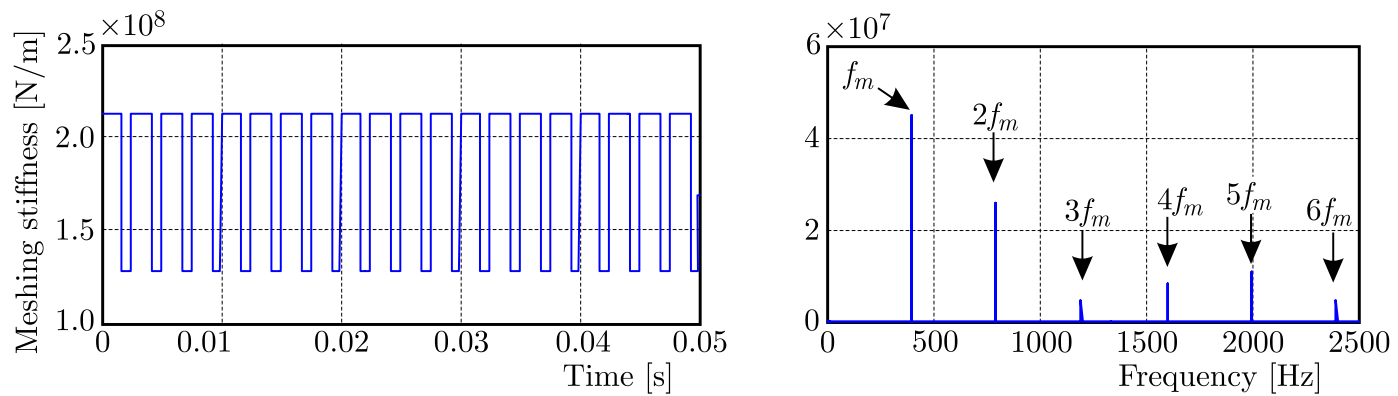

Fig. 4. Gear mesh stiffness for healthy case

The healthy configuration can be described by a step function with no phase and amplitude modifications. If the contact ratio is less than two, the minimum value corresponds to the mesh stiffness of one pair of teeth in contact, whereas the maximum value being twice the minimum amplitude corresponding to two pairs of teeth in contact.

\subsection{Disc brake modelling}

The braking of the system is done by a disc brake system rigidly assembled with pinion 21. Composed mainly of the disc and two braking plates, the pair of brake pad assemblies is pressed 
against the disc in order to generate a frictional torque and, consequently, to slow down its rotation (Fig. 5). The braking torque $C_{b r}$ can be expressed by the Coulomb model and it is considered as an externally applied force in the equations of motion of the studied system.

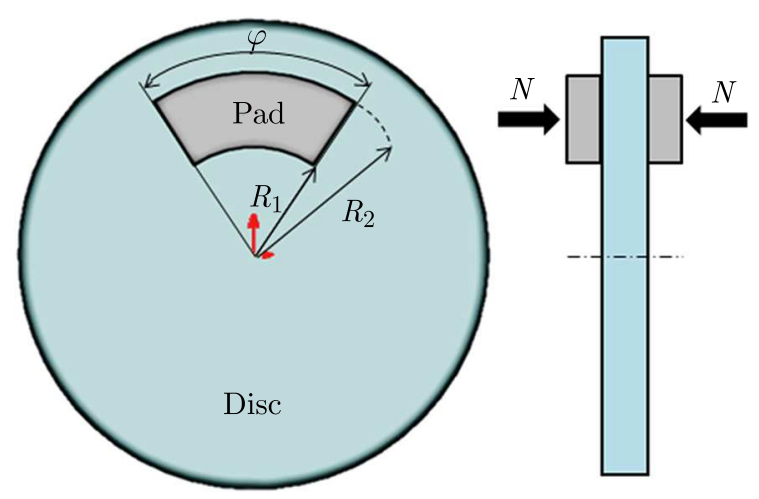

Fig. 5. Disc brake model

The braking torque $C_{b r}$ can be expressed as follows

$$
C_{b r}=\frac{4 \mu N \varphi\left(R_{1}+R_{2}\right)}{6 \sin (\varphi / 2)}\left(1-\frac{R_{1} R_{2}}{\left(R_{1}+R_{2}\right)^{2}}\right)
$$

where $\mu$ is the friction coefficient of the pad, $N$ is the normal force, $\varphi$ is the pad opening angle and $R_{1}$ and $R_{2}$ are, respectively, the outer and inner radii of the pad.

\section{Equations of motion}

Lagrange's method has been used to formulate the nonlinear differential equation governing the drivetrain system with thirteen degrees of freedom, which is

$$
\mathbf{M}_{G} \ddot{\mathbf{q}}+\mathbf{C} \dot{\mathbf{q}}+\mathbf{K}_{G} \mathbf{q}=\mathbf{F}_{0}(t)+\mathbf{F}(q)
$$

where $\mathbf{q}$ is the vector of degrees of freedom defined by

$$
\mathbf{q}=\left[x_{1}, y_{1}, x_{2}, y_{2}, x_{3}, y_{3}, \theta_{1}, \theta_{2}, \theta_{3}, \theta_{4}, \theta_{12}, \theta_{21}, \theta_{d}\right]
$$

where $x_{i}$ and $y_{i}(i=1,2,3)$ are the bearing displacements. $\theta$ represents the angular displacement of the wheel and gears (12 and 21) along the direction $z$.

$\mathbf{M}_{G}$ is the global mass matrix and expressed by

$$
\mathbf{M}_{G}=\operatorname{diag}\left(m_{b 1}, m_{b 1}, m_{b 2}, m_{b 2}, m_{b 3}, m_{b 3}, I_{1}, I_{2}, I_{3}, I_{4}, I_{12}, I_{21}, I_{d}\right)
$$

$\mathbf{K}_{G}$ is the stiffness matrix and written as

$$
\mathbf{K}_{G}=\mathbf{K}_{e}(t)+\mathbf{K}
$$

where $\mathbf{K}_{e}(t)$ is the linear time varying mesh stiffness matrix and $\mathbf{K}$ designates the constant stiffness matrix of the bearing and shafts and written as

$$
\mathbf{K}=\operatorname{diag}\left(\mathbf{K}^{b}, \mathbf{K}^{s h}\right)
$$


where the matrices $\mathbf{K}_{b}$ and $\mathbf{K}_{s h}$ are expressed by

$$
\begin{aligned}
\mathbf{K}^{b}= & \operatorname{diag}\left(k_{x 1}, k_{y 1}, k_{x 2}, k_{y 2}, k_{x 3}, k_{y 3}\right) \\
\mathbf{K}^{s h}= & {\left[\begin{array}{ccccccc}
k_{12} & -k_{12} & 0 & 0 & 0 & 0 & 0 \\
-k_{12} & k_{12} & 0 & 0 & 0 & 0 & 0 \\
0 & 0 & K_{1} & -K_{1} & 0 & 0 & 0 \\
0 & 0 & -K_{1} & K_{1}+K_{2} & -K_{2} & 0 & 0 \\
0 & 0 & 0 & -K_{2} & K_{2} & 0 & 0 \\
0 & 0 & 0 & 0 & 0 & K_{3} & -K_{3} \\
0 & 0 & 0 & 0 & 0 & -K_{3} & K_{3}
\end{array}\right] }
\end{aligned}
$$

$K_{i}$ is the torsional stiffness of the shaft $i$.

The gear mesh stiffness matrix can be written as follows

$$
\mathbf{K}(t)=\mathbf{L}_{\delta}^{\mathrm{T}} \mathbf{L}_{\delta} k(t)
$$

where $k(t)$ is the total meshing stiffness. $\mathbf{L}_{\delta}$ is the teeth deflection and can be written as follows

$$
\mathbf{L}_{\delta}=\left[0,0, s_{1}, s_{2},-s_{1},-s_{2}, 0,0,0,0, s_{3}, s_{4}, 0\right]
$$

The constants of deflection $s_{i}, i=1,2,3,4$ are given in Table 1 , where $\alpha$ is the pressure angle. The base radii of pinions 12 and 21 are respectively $R_{b 12}$ and $R_{b 21}$.

Table 1. Coefficients $s_{i}$ of $K(t)$

\begin{tabular}{|c|c|c|c|}
\hline$s_{1}$ & $s_{2}$ & $s_{3}$ & $s_{4}$ \\
\hline \hline $\sin \alpha$ & $\cos \alpha$ & $R_{b 12}$ & $R_{b 21}$ \\
\hline
\end{tabular}

$\mathbf{C}$ is the damping matrix proportional to the mass and stiffness matrix

$$
\mathbf{C}=\lambda \mathbf{M}_{G}+\eta \widetilde{\mathbf{K}}_{G}
$$

where $\widetilde{\mathbf{K}}_{G}$ is the average rigidity matrix, independent of time. $\lambda$ and $\eta$ are determined experimentally.

The excitation vector is expressed by

$$
\begin{aligned}
& \mathbf{F}(t, \dot{q})=\left[F_{T x 2 / 3}, F_{T y 2 / 3},-F_{T x 2 / 3},-F_{T y 2 / 3}, 0,0, T_{e}(t)-T_{f}\left(\dot{\delta}_{3}\right),\right. \\
& \left.\quad-T_{f}\left(\dot{\delta}_{2}\right), T_{f}\left(\dot{\delta}_{2}\right)+T_{f}\left(\dot{\delta}_{3}\right), 0,0,0,-T_{d}+C_{b r}\right]
\end{aligned}
$$

The terms $F_{T x 2 / 3}$ and $F_{T y 2 / 3}$ are tangential forces applied by the second block on the third block along the direction $x$ and $y$

$$
F_{T x 2 / 3}=-\mu_{D} P A \frac{\dot{x}_{3}-\dot{x}_{2}}{\left\|\dot{x}_{3}-\dot{x}_{2}\right\|} \quad F_{T y 2 / 3}=-\mu_{D} P A \frac{\dot{y}_{3}-\dot{y}_{2}}{\left\|\dot{y}_{3}-\dot{y}_{2}\right\|}
$$

\section{Effect of damage in spur gear on the dynamic response}

A elements in various mechanical driveline systems have a specific vibration pattern that depends on work conditions and construction. Variation in the vibration pattern in any position of the system indicates the initiation of defect in the machine. The main objective of vibration analysis is to identify the condition of the drivetrain to distinguish healthy and defected geared systems and to identify the defective components. In this part, the effect of local damage and profile error on the geared system (gear 12) on the dynamic behavior of different components of the drivetrain will be investigated. 


\subsection{Effect of local damage in spur gear on the dynamic response}

The main purpose of this Section is to investigate the effects of introduction of the local damage into the geared system on the dynamic response. In the presence of a local damage defect, the meshing stiffness variations give important information about the dynamic behavior of the system. For the modeling of the local gear defect, three approaches have been used in the literature. The first approach is based on the fact that tooth wear can cause loss of contact due to deviation of the ideal profile, resulting in a periodic decrease in the tooth stiffness. The second approach assumes that wear causes reduction of the driving ratio from where it delays the phase where two pairs of teeth come into contact with a decrease in the amplitude of the maximum stiffness associated with this phase. The third approach consists in reducing the two extreme values of the stiffness in addition to the reduction of the driving ratio at the level of the period affected by this defect. The first two approaches are the most used in the research work.
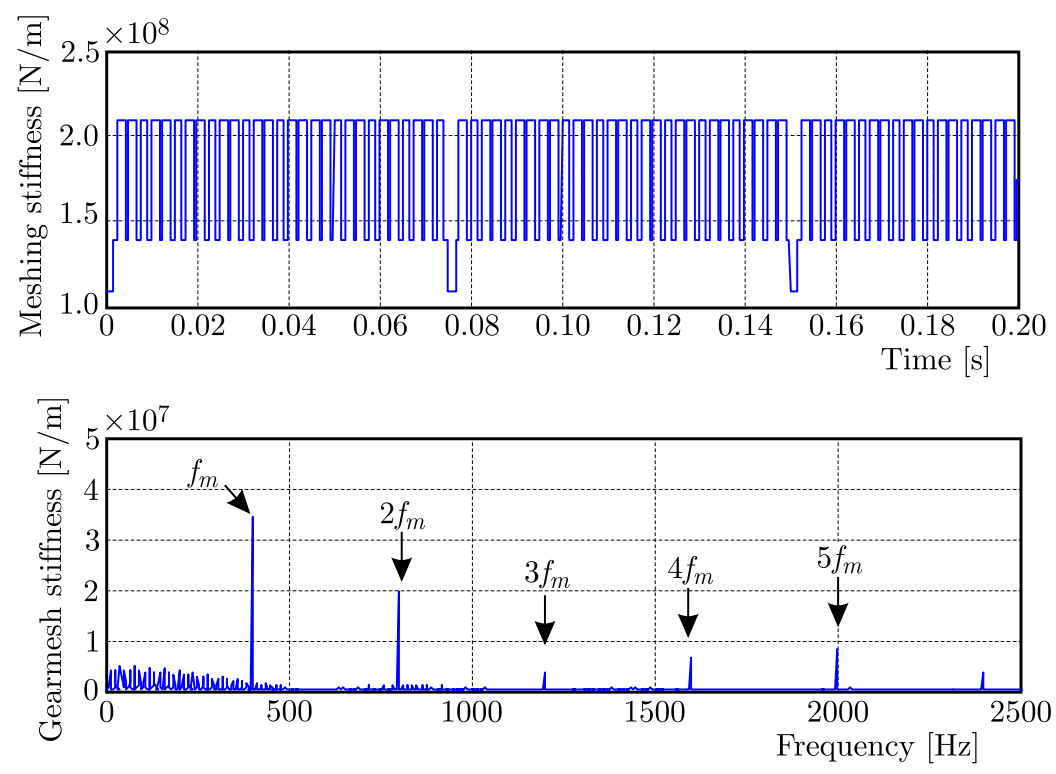

Fig. 6. Gear mesh stiffness for local defect pinion tooth: (a) time response, (b) corresponding spectrum

The tooth damage is modeled by a loss in the mesh stiffness, and it is shown in Fig. 6a. In the presence of a local defect, the corresponding spectrum (Fig. $6 \mathrm{~b}$ ) shows that the effect of defect on the vibration signal is the amplitude modulation around the meshing frequency $f_{m}$ and a comb lines whose pitch corresponds to the pinion rotation frequency $f_{p}$.

Using the parameter values illustrated in Table 2 , the velocity vibration signals of the second bearing along the $y$ direction $\left(\dot{y}_{2}\right)$ and the speed time response $\left(\dot{\theta}_{12}\right)$ of defected gear 12 in the two cases; for both the perfect working condition and in the presence of the local defect on gear 12 are shown in Figs. 7a and 7b, respectively. The local damage occurred on the gear tooth and generated a short duration impulsive signal. The impulse period is $0.075 \mathrm{~s}$, which is equal to the rotational frequency of the defected wheel (gear 12). The local impulse in the vibration signal is periodic and repeated every rotation of gear 12 resulting an additional amplitude and phase modulation.

In the following, we are interested in studying the effects of faults on each subsystem and in selecting different terms. In the gearbox, we study variation of the dynamic transmission error (DTE) which is defined in Eq. (4.1). From the literature, it seems clear that the transmission error represents an interesting indicator of the vibratory behavior of a gear train. On the other hand, deformations of bodies, teeth as well as other components such as shafts and bearings are encompassed in this concept. To investigate the clutch dynamic behavior, tangential forces 
Table 2. Values of parameters for numerical studies of the system

\begin{tabular}{|l|l|}
\hline Torque excitation $T_{p}, T_{p m}[\mathrm{Nm}]$ & $T_{p}=300, T_{p m}=250$ \\
\hline Engine speed $\Omega_{e}[\mathrm{rpm}]$ & $\Omega_{e}=800 \mathrm{rpm}$ \\
\hline Masses $m_{b 1}, m_{b 2}, m_{b 3}[\mathrm{~kg}]$ & $m_{b 1}=0.5, m_{b 2}=3, m_{b 3}=10$ \\
\hline Inertias $I_{1}, I_{2}, I_{3}, I_{4}\left[\mathrm{~kg} \mathrm{~m}{ }^{2}\right]$ & $I_{1}=0.2, I_{2}=12 \cdot 10^{-3}, I_{3}=8 \cdot 10^{-4}, I_{4}=2 \cdot 10^{-4}$ \\
\hline Torsional stiffness $[\mathrm{Nm} / \mathrm{rad}]$ & $k_{12}=8 \cdot 10^{4}, K_{1}=K_{2}=K_{3}=3 \cdot 10^{5}$ \\
\hline Bearing stiffness $[\mathrm{N} / \mathrm{m}]$ & $k_{x i}=k_{y i}=10^{8}, i=1,2,3$ \\
\hline Number of teeth & $Z_{12}=30, Z_{21}=45$ \\
\hline Normal load $[\mathrm{N}]$ & $N=1000$ \\
\hline
\end{tabular}

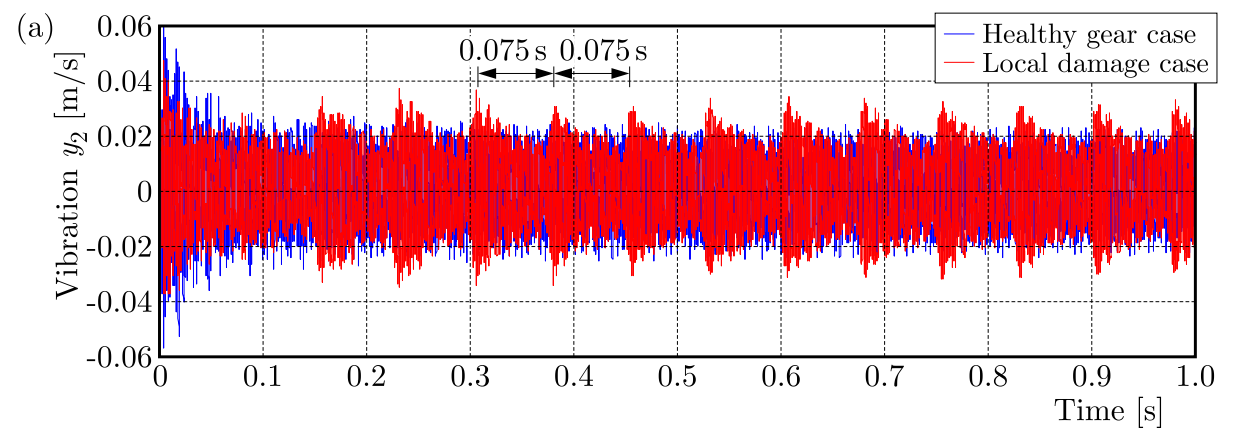

(b)

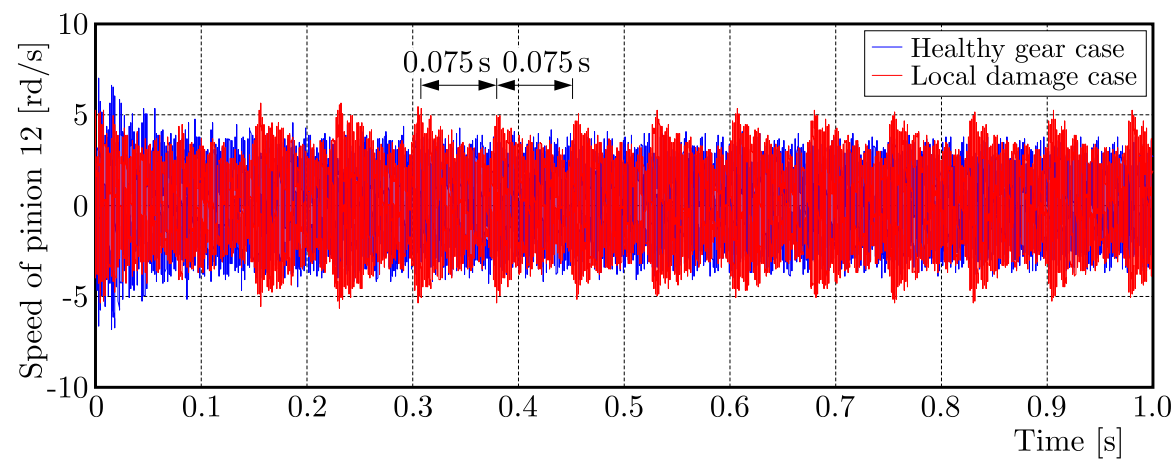

Fig. 7. Time response of the second bearing speed along the $y$ direction (a) and of the pinion speed (b); red line - case with the defect, blue line - case without the defect

applied to the friction disc are taken into account which allows us to give an idea on the torque transmitted by the clutch. The disc brake is treated by studying disc vibration since the disc undergoes the most important vibration

$$
\mathrm{DTE}=\theta_{12}-\frac{Z_{21}}{Z_{12}} \theta_{21}
$$

where $Z_{12}$ and $Z_{21}$ represents, respectively, the teeth number of pinions 12 and 21.

On the temporal signal, the dynamic transmission error DTE magnitude does not give a significant change with the presence of a fault. It is hard to detect clear symptoms of any error in the system from the time domain only, especially when the system is complex. The frequency domain can be used and it is the most popular approach to diagnosis of defects of the gear. The spectrum of the DTE in two cases, healthy and defected pinions, are shown in Fig. 8. In the frequency spectrum in Fig. 9, two frequencies appear: frequency of the meshing stiffness $f_{m}$ and its harmonics, and frequency of rotation of the pinion $f_{p}$. The spectrum shows peaks corresponding to the harmonic frequencies $f_{p}$. The other peaks represent the natural frequencies of the system.

The dynamic behavior of the clutch affects not only the dynamics of the drivetrain system but also the transfer of the excitation to drive wheels. Figure 9 shows the temporal signals 


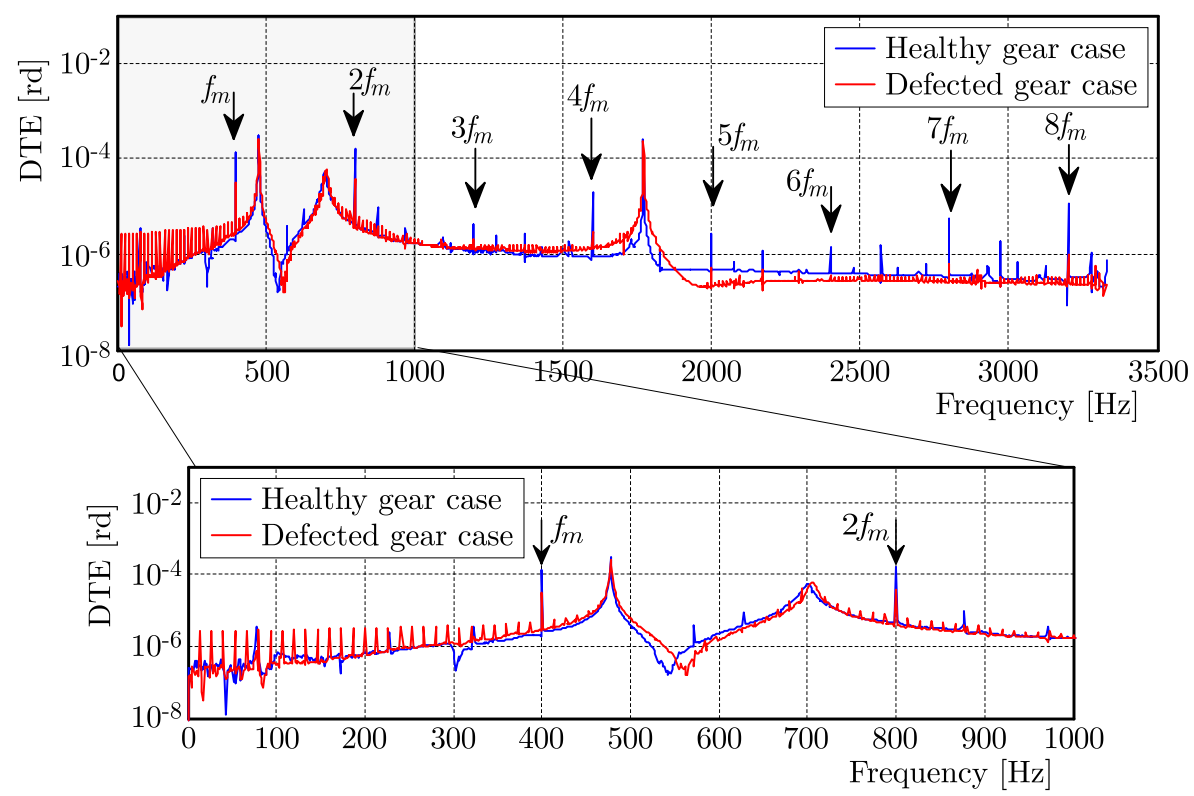

Fig. 8. Spectrum of the dynamic transmission error; red line - case with the defect, blue line - case without the defect
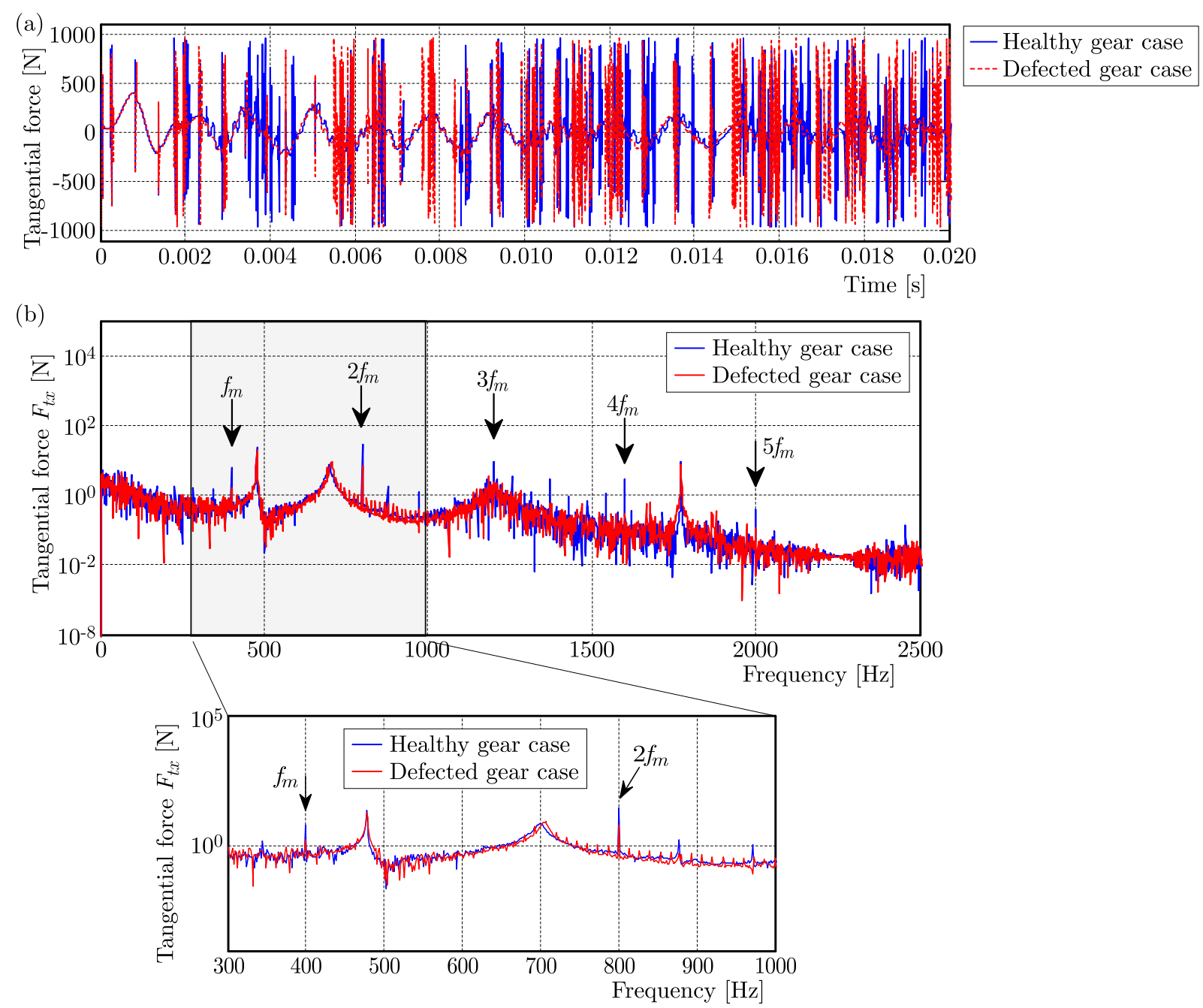

Fig. 9. Nonlinear tangential force $F_{T x 2 / 3}$ along the $x$ direction: (a) time response, (b) corresponding spectrum; red line - case with the defect, blue line - case without the defect 
and the frequency spectrum of the clutch tangential force $F_{T x 2 / 3}$ along the $x$ direction. In the case without the defect, the spectra show peaks around the meshing frequency $f_{m}$ and some eigenfrequencies (in the frequency band $300 \mathrm{~Hz}$ to $1000 \mathrm{~Hz}$ ). The dynamic model in this case is excited only by the periodic time varying meshing stiffness (internal excitation). In the presence of local damage, there appears in the spectrum a new peak at the frequency $f_{p}$, which corresponds to the frequency of rotation of gear 12 .

Figure 10 shows spectra of the two tangential forces applied by the second block on the third block and defined by Eq. (3.10) in the case with the defect. In the presence of the gear fault, the same remarks are available for $F_{T y 2 / 3}$ with $F_{T x 2 / 3}$, and we can interpret that the force amplitude along the $y$ direction is slightly higher than the force along the $x$ direction.

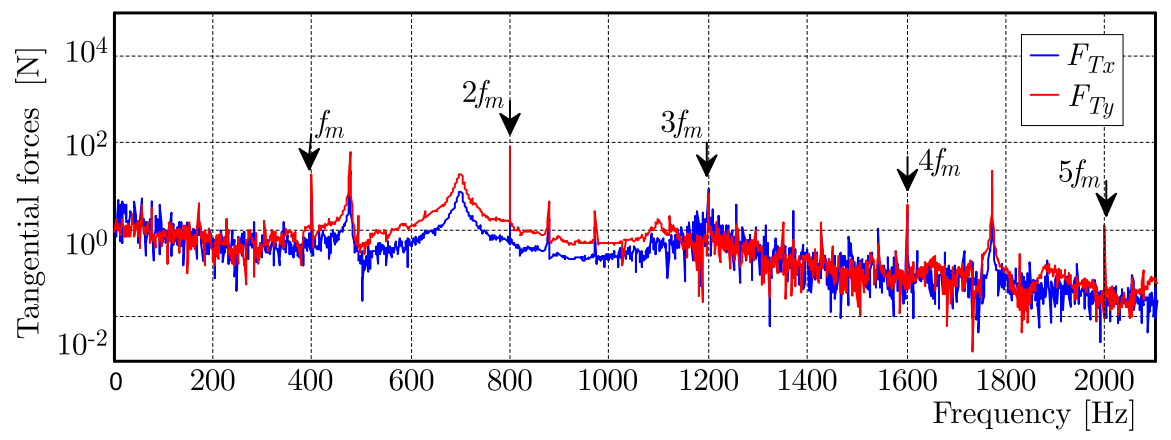

Fig. 10. Nonlinear tangential forces along $x$ and $y$ directions; blue line $-F_{T x}$, red line $-F_{T y}$

Figure 11 represents the frequency response of linear rotation of the brake disc. We can clearly see the presence of several peaks corresponding to the mesh frequency with their harmonic and natural frequency of the system. Also, other peaks can be shown which represent the frequency of pinion rotation $f_{p}$. This is due to local damage of the gearbox.

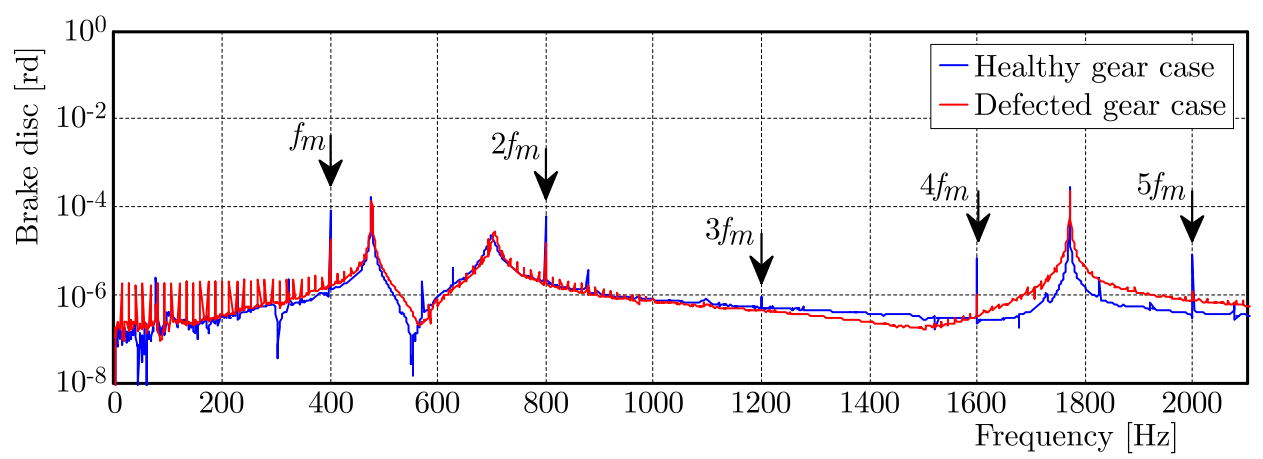

Fig. 11. Spectrum of the brake disc vibration; red line - case with the defect, blue line - case without the defect

\subsection{Effect of the profile error in spur gear on the dynamic response}

In gear systems, the profile defect is characterized by deviation of shape between the ideal profile of a tooth and the theoretical profile in the form of a circle. This defect often comes from the process of machining of teeth. The profile defect is introduced into equations of motion by adding the exciting term of the displacement type denoted by $e_{12}$ at the level of tooth deflection on the line of action. This defect is modelled in Fig. 12. 


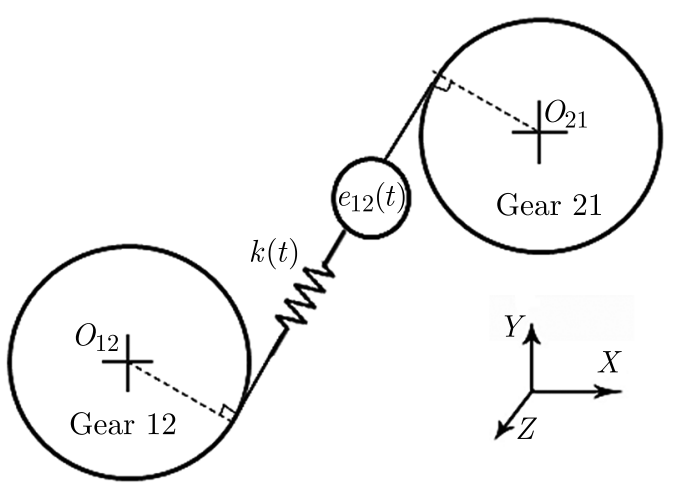

Fig. 12. Profile error modelling

The profile error is assumed to be uniform over all teeth. Transmission error due to this defect can be assimilated by a function which varies periodically with the meshing period $T_{m}$ as

$$
e_{12}(t)=e_{12}+\sum_{i=1}^{\infty} e_{12} \sin \left(2 \pi i f_{m} t\right)
$$

where $e_{12}, f_{m}$ and $t$ are, respectively, the error profile amplitude, meshing frequency and time variation.

The introduction of this excitation into the equations of motion gives rise to a supplementary force of external origin given by

$$
\mathbf{F}_{\text {sup }}=k(t) e_{12}(t) \mathbf{L}_{\delta}
$$

The profile error of the gear with the amplitude $e_{12}=50 \mu \mathrm{m}$ will amplify the amplitude of the transmission error. This result is ilustrated in Fig. 13a. The logarithmic representation of the spectrum shows that the meshing frequency is amplified and will be clear in the frequency shape with detecting other peaks in the signal (Fig. 13b).

(a)

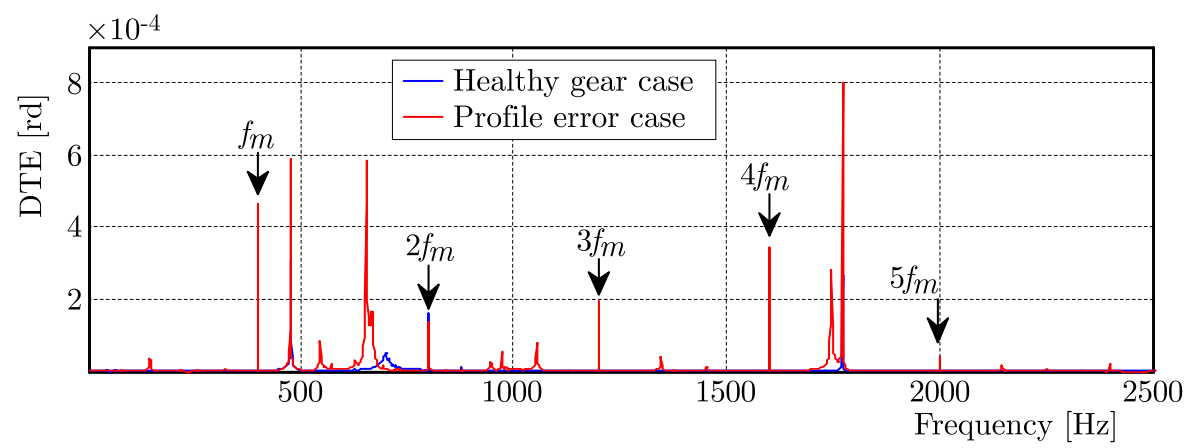

(b)

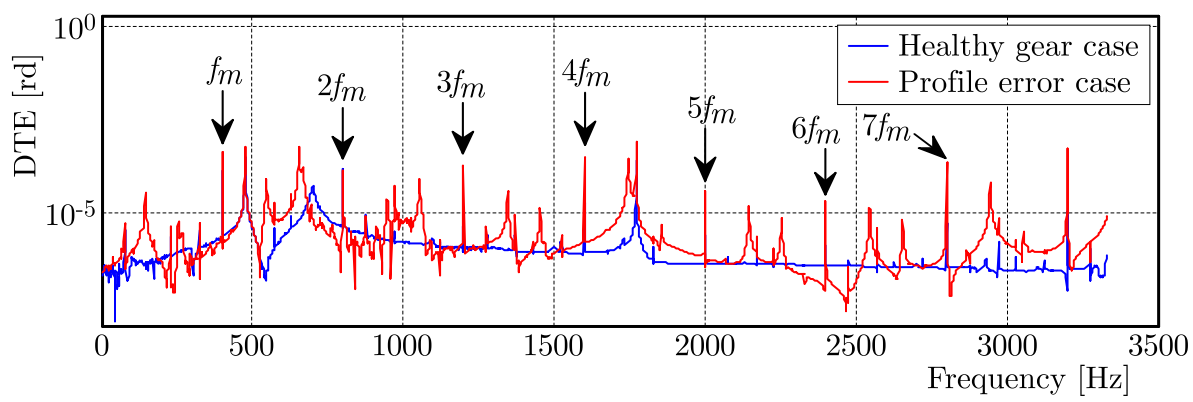

Fig. 13. Spectrum of the dynamic transmission error: (a) linear scale, (b) logarithmic scale; red line - case with the profile error, blue line - case without the defect 
These results are validated with the spectrum frequency of the tangential force of the clutch and brake disc vibration in Figs. 14 and 15. The frequency level increases but the signal will be more complex and difficult to treat especially with the logarithmic representation.

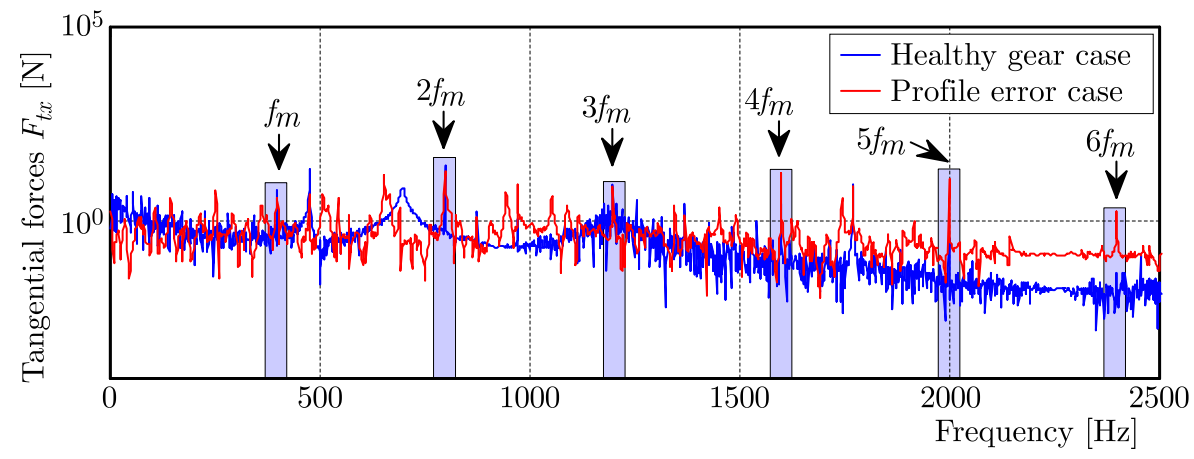

Fig. 14. Spectrum of nonlinear tangential forces $F_{T x 2 / 3}$ along the $x$ direction; red line - case with the profile error, blue line - case without the defect

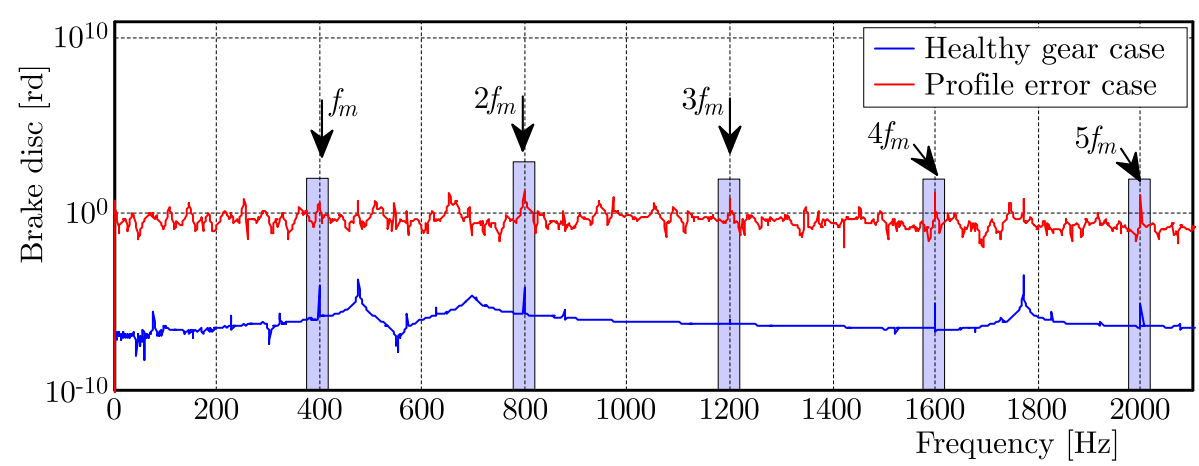

Fig. 15. Spectrum of brake disc vibration; red line - case with the profile error, blue line - case without the defect

\subsection{Effect of the two gear defects on the dynamic response}

Figures 16, 17 and 18 represent the frequency response on the dynamic components of the transmission error, clutch tangential force and brake disc torsional vibration in the two case studies. We conclude that the effects of the two faults are clear in the spectrum representation, especially in the DTE signal. The local damage detected by appearing as side bands of the fundamental frequency and the profile error can be diagnosed by amplification of the frequency. Another interpretation can be noticed that the peaks due to the local defect are also amplified.

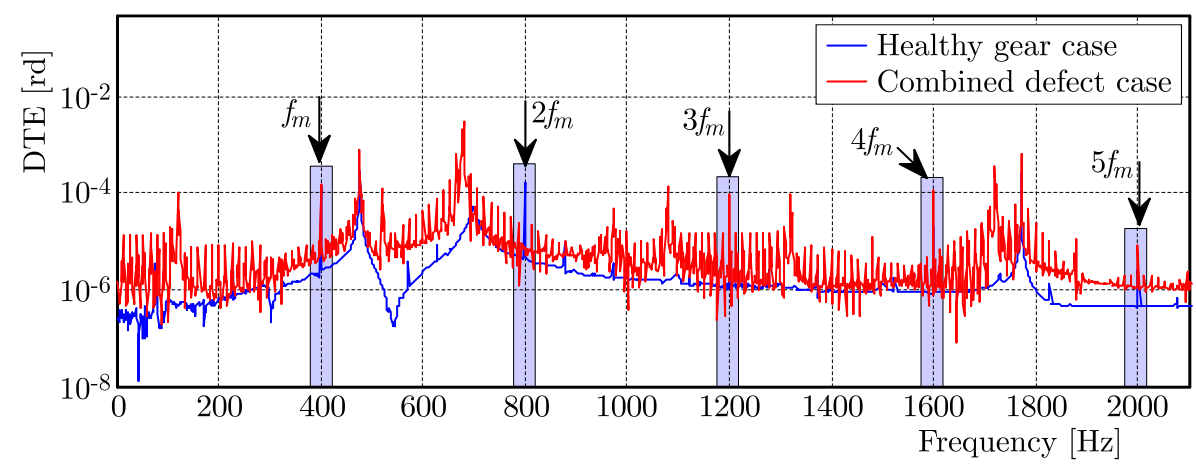

Fig. 16. Spectrum of the dynamic transmission error; red line - with two defects, blue line - without defects 


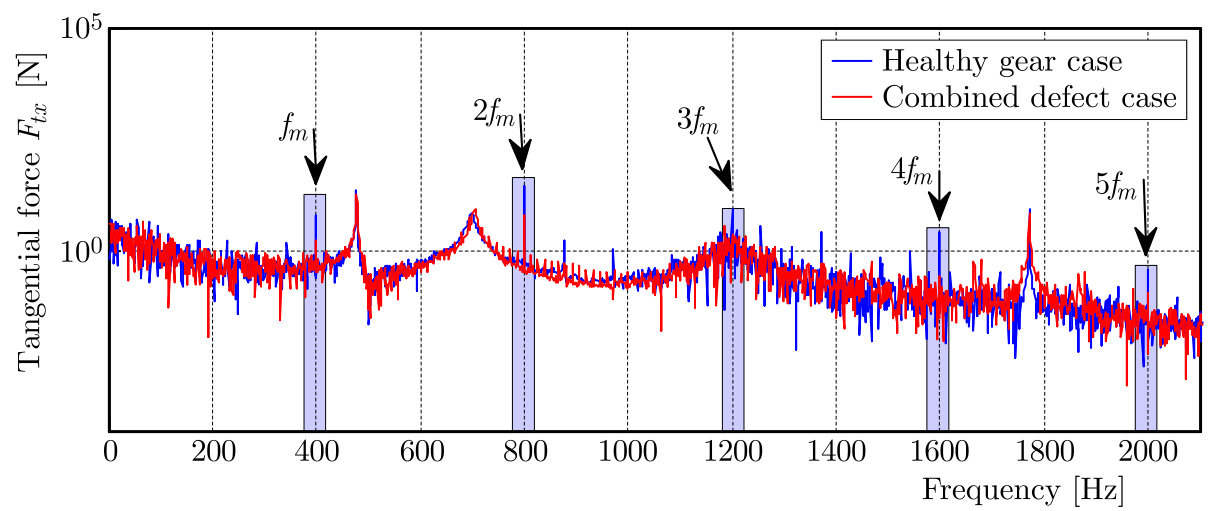

Fig. 17. Spectrum of the tangential force $F_{T x 2 / 3}$ along the $x$ axis; red line - with two defects, blue line - without defects

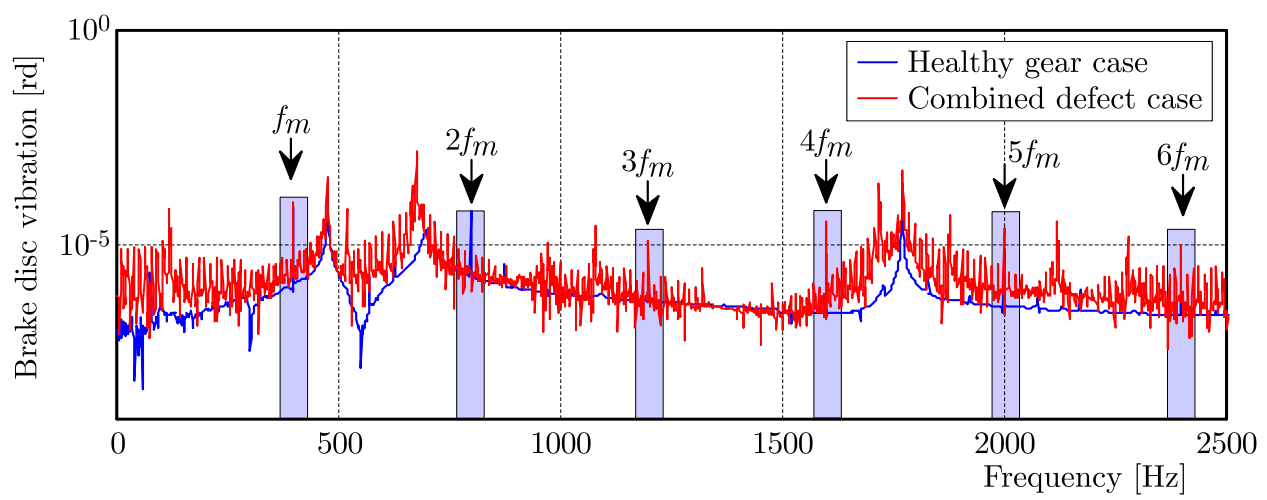

Fig. 18. Spectrum of brake disc vibration; red line - with two defects, blue line - without defects

\section{Conclusion}

The purpose of this research work is to develop a nonlinear dynamic model of a vehicle drivetrain system, introduce two typical defects and investigate the effect of these faults on the dynamic response of the system. To achieve this outcome, the developed model with thirteen degrees of freedom that includes engine excitation, clutch, gearbox and disc brake is detailed and the differential governing equation is solved by the Runge Kutta integration method. The dynamic behavior of both the faultless drivetrain (without defect), and the mechanism with the local damage and profile error of the gear is compared in time and frequency domains. In the faultless mechanism, the spectrum reveals peaks at the meshing frequencies $f_{m}$ and the dominant eigenfrequency. In the presence of the local defect, amplitude modulation occurs in the time signal of the bearing and the gear. From the FFT spectrum, it is observed that the effect of the local fault appears in the frequency domain signal as sidebands of the pinion rotation frequency. Introducing the profile error, the signal vibratory level increases. These results are verified in the literature. Finally, the two defects are integrated together and despite the complexity of the system, the two faults in the frequency representation can be detected. Comparison of these frequency spectra at the healthy and defected condition of the gear transmission reflects significant symptoms of local damage and profile errors on the vibrational behavior of the system.

\section{References}

1. Abouel-Seoud S., Ibrahim A., Mohamed K., 2012, An experimental study on the diagnostic capability of vibration analysis for wind turbine planetary gearbox, International Journal of Modern Engineering Research, 2, 3, 667-675 
2. Aherwar A., Khalid Md.S., 2012, Vibration analysis techniques for gearbox diagnostic: a review, International Journal of Advanced Engineering Technology, 3, 2, 4-12

3. Ahmed I., 2011, Analysis of disc brake squeal using a ten-degree-of-freedom model, International Journal of Engineering, Science and Technology, 3, 142-155

4. Bemporad A., Borrelli F., Glielmo L., Vasca F., 2001, Optimal piecewise-linear control of dry clutch engagement, IFAC Workshop Advances in Automotive Control, 33-38

5. Brancati R., Ernesto R., Riccardo R., 2007, An analysis of the automotive driveline dynamic behaviour focusing on the influence of the oil squeeze effect on the idle rattle phenomenon, Journal of Sound and Vibration, 303, 858-872

6. Chatri F., Baccar W., Abbes M.S., Haddar M., 2008, Effect of spalling or tooth breakage on gear mesh stiffness and dynamic response of a one-stage spur gear transmission, European Journal of Mechanics-A/Solids, 27, 691-705

7. Divandari M., Aghdam B.H., Barzamini R., 2012, Tooth profile modification and its effect on spur gear pair vibration in presence of localized tooth defect, Journal of Mechanics, 28, 373-381

8. Driss Y., FAKhfakh T., Haddar M., 2007, Effect of eccentricity on a clutch system under a harmonically varying normal load, Journal of Failure Analysis and Prevention, 7, 127-136

9. Fakhfakh T., ChaAri F., Haddar M., 2005, Numerical and experimental analysis of a gear system with teeth defects, The International Journal of Advanced Manufacturing Technology, 25, $542-550$

10. Fakhfakh T., Walha L., Louati J., Haddar M., 2006, Effect of manufacturing and assembly defects on two-stage gear systems vibration, The International Journal of Advanced Manufacturing Technology, 29, 1008-1018

11. Fosberry R.A.C., Holubecki Z., 1961, Disc Brake Squeal: its Mechanism and Suppression, Motor Industry Research Association

12. Gaillard C.L., Singh R., 2000, Dynamic analysis of automotive clutch dampers, Applied Acoustics, 60, 399-424

13. Ghorbel A., Abdennadher M., Zghal B., Walha L., Haddar M., 2017, Modal analysis and dynamic behavior for analytical drivetrain model, Journal of Mechanics, DOI: 10.1017/jmech.2017.23

14. HowArd I., Jia S., WANG J., 2011, The dynamic modelling of a spur gear in mesh including friction and a crack, Mechanical Systems and Signal Processing, 15, 831-853

15. Khabou M.T., Ksentini O., Jarraya A., Abbes M.S., Chaari F., Haddar M., 2014, Influence of disk brake friction on the dynamic behaviour of a directly coupled spur gear transmission, Multidiscipline Modeling in Materials and Structures, 10, 146-162

16. Kim W., Yoo H.H., Chung J., 2010, Dynamic analysis for a pair of spur gears with translational motion due to bearing deformation, Journal of Sound and Vibration, 329, 4409-4421

17. Kobra H., Hojat A., Omid M., 2010, Adaptive vibration condition monitoring techniques for local tooth damage in gearbox, Modern Applied Science, Canadian Center of Science and Education, 4

18. Krak M., Jason D., Singh R., 2015, Development of a non-linear clutch damper experiment exhibiting transient dynamics, SAE Technical Paper Series, 01-2189

19. Mashadi B., Milad B., 2015, Driveline oscillation control by using a dry clutch system, Applied Mathematical Modelling, 39, 6471-6490

20. Rincon A.F.D., Viadero F., Iglesias M., Juan A.D., Garcia P., Sancibrian R., 2012 , Effect of cracks and pitting defects on gear meshing, Proceedings of the Institution of Mechanical Engineers, Part C: Journal of Mechanical Engineering Science, 226, 2805-2815

21. Saleh A., Krak M., Jason D., Singh R., 2015, Development of refined clutch-damper subsystem dynamic models suitable for time domain studies, SAE Technical Paper Series, 01-2180 
22. Templin P., Egardt B., 2009, An LQR torque compensator for driveline oscillation damping, 18th IEEE International Conference Control Applications, 352-356

23. WAlha L., FAKhfakh T., HAdDAR M., 2009, Nonlinear dynamics of a two-stage gear system with mesh stiffness fluctuation, bearing flexibility and backlash, Mechanism and Machine Theory, 44, 1058-1069

24. Walha L., Driss Y., Khabou M.T., Fakhfakh T., Haddar M., 2011, Effects of eccentricity defect on the nonlinear dynamic behavior of the mechanism clutch-helical two stage gear, Mechanism and Machine Theory, 46, 986-997

25. Wu H., Guangqiang W., 2016, Driveline torsional analysis and clutch damper optimization for reducing gear rattle, Shock and Vibration, 11, 1-24

26. Xue-Lai L., Wen-Bin S., Xinguian J., Waizuddin A., 2016, Vibration isolation analysis of clutches based on trouble shooting of vehicle accelerating noise, Journal of Sound and Vibration, 382, 84-99

Manuscript received May 25, 2017; accepted for print December 4, 2017 